\title{
Reliability and Validity of the Farsi Version of the Somatosensory Amplification Scale
}

\author{
Alireza Aghayousefi, ${ }^{1}$ Mohammad Oraki, ${ }^{1}$ Narges Mohammadi, ${ }^{2, *}$ Valiyollah Farzad, ${ }^{3}$ and \\ Hammed Daghaghzadeh $^{4}$ \\ ${ }^{1}$ Department of Psychology, Payame Noor University, Tehran, IR Iran \\ ${ }^{2}$ Psychosomatic Research Center, Isfahan University of Medical Sciences, Isfahan, IR Iran \\ ${ }^{3}$ Department of Psychology, Kharazmi University, Tehran, IR Iran \\ 4 Integrative Functional Gastroenterology Research Center, Isfahan University of Medical Sciences, Isfahan, IR Iran \\ *Corresponding author: Narges Mohammadi, Psychosomatic Research Center, Isfahan University of Medical Sciences, Isfahan, IR Iran. E-mail: moha5190@yahoo.com
}

Received: October 16, 2014; Revised: November 15, 2014; Accepted: December 20, 2014

\begin{abstract}
Background:The somatosensory amplification scale(SSAS) is a 10-item self-report instrument designed to assess a tendency to experience normal somatic and visceral sensations as intense, noxious, and disturbing.

Objectives:The present study investigated the reliabilityand validity of the SSAS, developed by Barskyetal.(1988), in the Iranian population. Materials and Methods: The study was carried out on 240 patients with functional gastrointestinal disorders and 30 healthy persons selected by convenience sampling from 2013 to 2014. The patients completed the SSAS, the somatization subscale of the symptom checklist90-revised (SCL-90-R som), and the modified somatic perception questionnaire (MSPQ), whereas the healthy persons completed just the SSAS.

Results: Exploratory factor analysis indicated that the one-factor solution, accounting for $29.42 \%$ of the variance, explained that the SSAS items were represented by one global dimension. The SSAS had acceptable internal consistency $(\alpha=0.78)$ and good test-retest reliability $(r=0.80)$. The item-to-scale correlations varied from 0.17 to 0.55. Item 2 had the lowest item-total score correlation $(r=0.17)$, and the $\alpha$ coefficient for the SSAS exceeded when this item was deleted. The convergent validity of the SSAS with somatization was shown with a significant correlation between the SSAS, SCL-90-R som $(r=0.36)$, and MSPQ scores $(r=0.52)$. Discriminant validity analysis showed no significant difference in the SSAS between the patient and control groups $(\mathrm{P}>0.05)$ and non-specificity of the SSAS for patients.

Conclusions: In sum, the SSAS has acceptable reliability and validity for the Iranian population and the scale measures the same the original scale, namely somatosensory amplification.
\end{abstract}

Keywords: Functional Gastrointestinal Disorders; Reliability; Somatoform Disorders; Somatosensory Amplification; Validity

\section{Background}

The somatosensory amplification scale (SSAS) is a 10item self-report instrument designed to assess a tendency to experience normal somatic and visceral sensations as intense, noxious, and disturbing (1).

The concept of somatosensory amplification involves self-examination, increased attention, and vigilance to unpleasant somatic sensations, a predisposition to observe weak and rare somatic sensations and a tendency to interpret them as dangerous signs of disease without any rational cause (2). Therefore, somatosensory amplification may have 3 main components: 1 ) hyper vigilance towards unpleasant bodily sensations; 2) tendency to select and focus on certain relatively weak or infrequent sensations; and 3) tendency to appraise ambiguous or vague visceral and somatic sensations as abnormal and symptomatic of disease, rather than considering them to be normal (3).

The construct of somatic amplification is useful for the comprehension of functional somatic symptoms such as those seen in somatoform disorders, especially hypochondriasis $(2,4,5)$. Somatosensory amplification is important even in the organic symptoms of serious medical diseases because it may explain the variability of the symptom reports in different individuals with the same organ pathology (6) such as chronic pain $(7,8)$, upper respiratory tract infections (9), migraine (10), and inflammatory bowel diseases (11).

Moreover, amplification may be a pathogenic mechanism in amplification disorders such as fibromyalgia (1) and functional gastrointestinal disorders such as irritable bowel syndrome (1) and functional dyspepsia $(12,13)$. Therefore, the concept of somatosensory amplification is noticeable since it gives a clearer understanding of why somatic symptoms are disproportionate to certain organ pathology (14). Moreover, it can highlight the pathogenic role of somatosensory amplification in somatization (15, 16) and currently, health anxiety process (17).

Copyright ( ) 2015, Mazandaran University of Medical Sciences. This is an open-access article distributed under the terms of the Creative Commons Attribution-NonCommercial 4.0 International License (http://creativecommons.org/licenses/by-nc/4.0/) which permits copy and redistribute the material just in noncommercial usages, provided the original work is properly cited. 
There are versions of the SSAS in several languages such as Spanish, Korean, Japanese, Italian, Turkish, Hungarian, and French (14), and the reliability and validity of these versions have been previously demonstrated.

The original version of the SSAS was developed by Barsky et al. (1), and it had good internal consistency (Cronbach's $\alpha=0.82$ ). While the test-retest reliability for the 10-item scale was 0.79 , its item-to-scale correlations varied between 0.31 and 0.66 and all were highly significant. Also, its item-to-item correlations varied from between not significant to 0.60 , but most were in the range of 0.35 and were highly significant (18). Although Barsky et al. (2) specified that the SSAS is only suitable for the samples of medical outpatients (2), more recently, an assessment of the validity and reliability of the French adaptation of the SSAS showed good internal consistency (Cronbach's $\alpha=0.81$ ) in a non-clinical population (14). Furthermore, the Turkish version of the SSAS was valid both for healthy individuals and patients and just reliable for healthy individuals (19).

Spinhoven and van der Does (19) observed that the SSAS is correlated with somatization (subscale of the symptom checklist 90 revised [SCL-90-R som]) and that this correlation is not dependent on gender, presence of physical illness, and depression scores. Muramatsu et al. (9) demonstrated that the SSAS is significantly associated with all somatic symptoms. According to Aronson et al. (15), the SSAS is not a measure for the evaluation of somatic sensitivity as an index of negative emotionality or general distress. Also, Wise and Mann (20) explained that the SSAS focuses particularly upon a perceptual style as a strong predictor of neuroticism. However, there are conflicts in the convergent and discriminant validity of this instrument, especially in somatization tendency. Also, the SSAS has been employed in more investigations as an instrument that assesses a single construct (i.e. the items are summed), and there has been little effort to investigate the factorial solution of the instrument.

Bridou and Aguerre (14) assessed the principal component and confirmatory factor analyses of the SSAS and suggested that the French version of the SSAS evaluates essentially a single and robust factor (somatosensory amplification).

We hypothesized that somatosensory amplification is an appropriate index of somatization propensity (convergent validity) and this construct is different between patients with functional somatic syndrome and healthy groups (discriminant validity).

\section{Objectives}

The present study aimed to evaluate the validity and reliability and especially the factorial solution of the Farsi version of the SSAS among patients with functional gastroenterological disorders.

\section{Materials and Methods}

\subsection{Participants and Procedure}

A forward and backward translation procedure was used for adapting the original American version of the SSAS in an Iranian patient population. First, one bilingual gastroenterologist (one of the authors of this article) translated the SSAS from English into Persian. This preliminary version was evaluated by 2 psychologists (the authors of this article), who discussed and corrected discrepancies and cultural adaptations. Then, a back-translation was carried out by a second professional translator to verify that our version matched the original version. Finally, the face validity of the SSAS was confirmed by 5 specialists (one gastroenterologist, one psychiatrist, and 3 psychologists, who work in the health psychology context).

The study was carried out on patients with functional gastrointestinal disorders diagnosed based on the ROMIII criteria referring to the outpatient psychosomatic clinic of Isfahan Medical University and the gastroenterologist's office (participating in this study) during a 10-month period from May 2013 to March 2014. Additionally, healthy persons were selected by convenience sampling. Totally, 240 patients with functional gastrointestinal disorders by filling 3 questionnaires (i.e. the SSAS, SCL-90-R som, and modified somatic perception questionnaire [MSPQ]), and 30 healthy persons by filling only one questionnaire (the SSAS) participated in the study.

The exclusion criteria were comprised of: 1) having serious medical diseases, 2) age less than 18 and more than 60 years, 3) not being fluent in the Persian language, 4) education less than high school, and 5) not giving informed consent.

The protocol of the study was approved by the ethics committee of Isfahan university medical sciences and was clarified for all the participants. Also, an informed consent was obtained from all the subjects.

\subsection{Measures}

The SSAS is a 10-item scale developed by Barsky et al. (1), and its validity and reliability have been previously demonstrated. This tool can be completed quickly (less than 10 minutes) and easily. Respondents are asked to rate the degree to which each assertion is characteristic of them in general on an ordinal scale from 1 (Not at all true) to 5 (Extremely true). In the original version, a total amplification score is obtained by adding the scores (ranging from 10 to 50). Most items describe a physical discomfort which does not indicate a disease. High scores indicate a greater tendency to intensify normal somatic sensations (14).

The somatization subscale of the SCL-90-R som was used to measure somatization. The scores for 20 items were added. Each self-reported item was rated on a scale of 0 (Not at all distressed by the item) to 4 (Extremely distressed by the item). The participants were asked to de- 
scribe the intensity of symptoms experienced during the previous week, with a higher score denoting a greater somatization propensity. The reliability and validity of the Farsi version of the SCL-90-R som have been previously validated in many investigations (21).

The MSPQ was administered to measure somatic arousal (heightened autonomic or somatic awareness). Such dysregulation may also be termed somatic anxiety or somatization. It can help identify somatic complaints that may be associated with psychological responses such as anxiety or depression. The MSPQ is a self-rating scale in which 13 somatic symptoms are rated on 4-point scales ( 0 not all; 3 extremely). The total score ranges from 0 to 39 , with higher scores indicating a greater risk of somatization. The participant is asked to rate how often he or she has experienced somatic symptoms during the past week. Investigations have shown that the MSPQ is psychometrically an instrument with adequate validity and reliability (22-24). Recently, good internal consistency (Cronbach's $\alpha=0.80$ ) of the Farsi version the MSPQ has been demonstrated (25).

\subsection{Statistical Analysis}

An exploratory factor analysis was used to reveal any latent variables within the SSAS that caused the manifest variables to covary. To ensure that the characteristics of the data set were suitable for the factor analysis to be conducted on the functional gastrointestinal disorders sample, the Kaiser-Meyer-Olkin (KMO) measure of sampling adequacy and the Bartlett test of sphericity (BTS) were conducted on the data. A maximum likelihood factor extraction procedure with varimax rotation was employed since this approach is particularly useful in extracting psychologically meaningful factors and because of the possibility that the extracted factors may be correlated. The screen test and multiple test runs were used to decide the number of meaningful factors that might be in the data set. The minimum loading of an item was determined at 0.35 .

Cronbach's $\alpha$ was used to investigate internal reliability, and 0.70 was considered as the minimum acceptable criterion of the instrument's internal reliability. The scale was given to 30 of the same functional gastrointestinal patients after one month to assess the test-retest consistency. The Pearson correlations were calculated to analyze the test-retest reliability and the relationships between somatosensory amplification and somatization in order to test the convergent validity. The analysis of variance (ANOVA) was used to measure the total scale of the discriminant validity for the groups in the study (patients and the healthy group).

SPSS 16.0 statistics software (SPSS Inc., Chicago, IL, USA) was used for the statistical analyses of the study data.

\section{Results}

\subsection{Descriptive Analysis}

The study was carried out on 240 patients with functional gastrointestinal disorders and 30 healthy persons.
The gender distribution was $81.7 \%$ female and $17.3 \%$ male in the patient group and $86 \%$ female and $14 \%$ male in the healthy group.

The age range of the patients was between 18 and 60 years, and the mean age was $35.14 \pm 11.72$ years. In the healthy group, the age range was between 21 and 54 years, and the mean age was $35.50 \pm 5.44$ years. Most of the subjects in both groups were college educated $(58.3 \%$ of the patients and $64 \%$ of the healthy persons). The diagnosis was irritable bowel syndrome in $87.5 \%$ of the patients and functional dyspepsia in $24 \%$.

The descriptive statistics of the SSAS scores in the patient group showed that mean and standard deviation of the SSAS scores were 34.68 and 5.82, respectively.

\subsection{Exploratory Factor Analysis}

The Kaiser measure of sampling adequacy yielded an index of 0.77 , and the BTS was significant $\left(\chi^{2}=372.5, \mathrm{df}=45\right.$, $\mathrm{P}<0.001)$. This preparatory analysis confirmed that the data distribution satisfied the psychometric criteria for the factor analysis to be performed.

To assess the underlying structure for the 10 items of the SSAS, factor extraction with maximum likelihood and varimax rotation were employed. The results of these analyses showed that the one-factor solution accounted for accumulatively $29.42 \%$ of the variance. In this model, the factor loadings of all the items except item 2 ("I am often aware of various things happening within my body") were significant and ranged from 0.35 to 0.68 . The 2 -factor solution accounted for accumulatively $41.20 \%$ of the variance and the 3 -factor solution, $51.77 \%$ of the variance. However, both model contained one low-loading and one factor with only 2 items. Moreover, item 2 was deleted again in these models (Table 1). The one-factor model denoted that the SSAS items were represented by one global dimension called somatosensory amplification.

\subsection{Reliability Analyses}

The reliability of the SSAS was evaluated by internal consistency (Cronbach's $\alpha$ ) and test-retest consistency.

The internal consistency, item total correlation, $\alpha$ if item deleted, and test-retest consistency values are given in Table 2. Cronbach's $\alpha$ value of the SSAS for the patients with functional gastrointestinal disorders was calculated to be 0.78 . When each item was considered, the item-to-scale correlations varied from 0.17 to 0.55 . It was notable that the correlation for item 2 was low $(r=$ 0.17 ). Also, the $\alpha$ coefficient for the SSAS exceeded when this item was deleted. Furthermore, the test-retest reliability for the total scale of the SSAS, after an interval of one month, was assessed in 30 of the same patients. According to the results in Table 2, the Farsi version of the SSAS was noted to be strongly correlated at a level of 0.80 and established to be consistent. 


\subsection{Convergent Validity Analyses}

The convergent validity of the SSAS with somatization was examined using 2 instruments (i.e. the somatization subscale of the SCL-90-R som and the MSPQ) in the patient group (Table 3). As was anticipated, the correlations between somatosensory amplification and somatization by the SCL-90-R som $(r=0.360, P=0.049)$, and MSPQ $(r=$
$0.522, \mathrm{P}=0.003$ ) were positively significant at a moderate level.

\subsection{Discriminant Validity Analysis}

For the discriminant validity, the total sores of the SSAS were compared between the patient and healthy groups. The results of the ANOVA analysis showed no significant difference between the two groups (Table 4).

\begin{tabular}{|c|c|c|c|c|c|c|}
\hline \multirow[t]{3}{*}{ Items } & \multicolumn{6}{|c|}{ Factor Loading } \\
\hline & \multirow[t]{2}{*}{$\begin{array}{l}\text { 1- Factor Model } \\
\text { Loadings }\end{array}$} & \multicolumn{2}{|c|}{$\begin{array}{l}\text { 2- Factor Model } \\
\text { Loadings }\end{array}$} & \multicolumn{3}{|c|}{ 3- Factor Model Loadings } \\
\hline & & I & II & $\mathbf{I}$ & II & III \\
\hline 1. I can't stand smoke, smog, or pollutants in the air. & 0.353 & 0.431 & & & & 0.842 \\
\hline \multicolumn{7}{|l|}{$\begin{array}{l}\text { 2. I am often aware of various things happening within my } \\
\text { body. }\end{array}$} \\
\hline 3. When I bruise myself, it stays noticeable for a long time. & 524 & & 0.562 & & 0.499 & \\
\hline 4. I sometimes can feel the blood flowing in my body. & 0.420 & 0.504 & & 0.553 & & \\
\hline 5. Sudden loud noises really bother me. & 0.651 & 0.494 & & & & 0.590 \\
\hline $\begin{array}{l}\text { 6. I can sometimes hear my pulse or my heartbeat throb in } \\
\text { my ear. }\end{array}$ & 0.688 & 0.755 & & 0.729 & & \\
\hline 7. I hate to be too hot or too cold. & 0.735 & 0.727 & & 0.751 & & \\
\hline 8. I am quick to sense the hung contractions in my stomach. & 0.591 & 0.433 & & 0.597 & & \\
\hline $\begin{array}{l}\text { 9. Even something minor, like an insect bite or a splinter, } \\
\text { really bothers me. }\end{array}$ & 0.641 & 0.718 & & 0.701 & & \\
\hline 10. I can't stand pain. & 0.425 & & 0.802 & & 0.809 & \\
\hline Explained variance, \% & 29.42 & 22.86 & 18.34 & 22.17 & 15.39 & 14.20 \\
\hline Cumulative variance, $\%$ & 29.42 & 41.20 & 51.77 & & & \\
\hline
\end{tabular}

Table 2. Internal Consistency Evaluation, Impact of Each Item on the Scale and Cronbach's Alpha Values if Item Deleted, and Test-Retest Consistency of the Somatosensory Amplification Scale $(n=240)^{\text {a }}$

\begin{tabular}{llc}
\hline Item No & Corrected Item-Total Correlation & Cronbach's Alpha if Item Deleted \\
\hline SSAS1 & 0.235 & 0.714 \\
\hline SSAS2 & 0.172 & 0.791 \\
\hline SSAS3 & 0.369 & 0.695 \\
\hline SSAS4 & 0.214 & 0.720 \\
\hline SSAS5 & 0.484 & 0.678 \\
\hline SSAS6 & 0.504 & 0.671 \\
\hline SSAS7 & 0.558 & 0.659 \\
\hline SSAS8 & 0.413 & 0.687 \\
\hline SSAS9 & 0.482 & 0.675 \\
\hline SSAS10 & 0.299 & 0.706 \\
\hline Internal consistency by Cronbach's alpha & & 0.78 \\
\hline Test-retest consistency by correlation & & 0.80 \\
\hline
\end{tabular}

\footnotetext{
a Abbreviation: SSAS, somatosensory amplification scale.
} 
Table 3. Correlations Between the Total SSAS, Symptom Checklist Revised Somatization Subscale (SCL-90-R Som), and the Modified Somatic Perception Questionnaire (MSPQ): 240 Patients With Functional Gastrointestinal Disorders ${ }^{\text {a }}$

\begin{tabular}{|c|c|c|c|}
\hline & SSAS & SCL-90-R som & MSPQ \\
\hline SSAS & 1 & $0.360^{b}$ & $0.522^{C}$ \\
\hline SCL-90-R som & & 1 & $0.788^{C}$ \\
\hline MSPQ & & & 1 \\
\hline
\end{tabular}

a Abbreviations: MSPQ, modified somatic perception questionnaire; SCL, symptom checklist; SSAS, somatosensory amplification scale.

b $\mathrm{P}<0.05$.

c $\mathrm{P}<0.01$.

\begin{tabular}{|c|c|c|c|c|c|c|c|c|c|}
\hline \multirow[t]{2}{*}{ ANOVA } & \multicolumn{9}{|c|}{ Test of Homogeneity of Variances } \\
\hline & Sum of Squares & df & Mean Square & $\mathbf{F}$ & Significance & Leven Statistic & $\mathrm{df}_{1}$ & $\mathbf{d f}_{2}$ & Significance \\
\hline Between groups & 61.341 & 1 & 61.341 & 1.734 & 0.189 & 1.423 & 1 & 268 & .234 \\
\hline Within groups & 9482.100 & 268 & 35.381 & & & & & & \\
\hline Total & 9543.441 & 269 & & & & & & & \\
\hline
\end{tabular}

a Abbreviations: ANOVA, analysis of variance; SSAS: somatosensory amplification scale.

\section{Discussion}

This investigation examined the Farsi version of the SSAS for validity and reliability.

The exploratory factor analysis of the SSAS demonstrated that a one-factor solution had the best fit for the data, accounting for approximately $29.42 \%$ of the variance. Our results support the hypothesis that the SSAS has a single dimension representing somatosensory amplification. Furthermore, it could be used to measure somatosensory amplification in patients with functional gastrointestinal disorders.

The findings are consistent with the original version of the SSAS, designed for the measurement of one factor, i.e. somatosensory amplification, in medical outpatients (2). Only a few studies have hitherto been conducted for the factor analysis of the SSAS, and most studies have used the scale as a one construct $(3,5-7,9,10,13,18)$. The exploratory and conformity factor analysis of the French version of the SSAS suggested that this scale evaluates essentially a single and robust factor (somatosensory amplification) and 2 kinds of somatic sensitivity (i.e. exteroceptive sensitivity and interoceptive sensitivity) (14). Therefore, the Farsi version of the SSAS also evaluates one factor as somatosensory amplification.

To test the reliability of the SSAS, internal consistency analysis and item total correlation and test-retest consistency were evaluated. Cronbach's $\alpha$ coefficient for internal consistency analysis was calculated to be 0.78 for the patients with functional gastrointestinal disorders. While Barsky et al. (2) established Cronbach's $\alpha$ to be 0.82 in medical outpatients, often studies have reported the similar internal consistency. Speckens et al. (26) demonstrated the $\alpha$ coefficient to be 0.77 in a hospital sample,
0.64 in patients in primary care, and 0.71 in the general population. Elsewhere, Gulec and Sayar (18) reported the $\alpha$ coefficient to be 0.76 in an outpatient group and 0.62 in a healthy group. Although the internal consistency in the present study was not very good, it can be considered acceptable.

The item total correlations were 0.17 - 0.55 in the patient group. It was also noted that item number 2 had the lowest correlation with the scale $(r=0.17)$ and the $\alpha$ coefficient of the SSAS increased when this item was deleted. This may be due to cultural differences or translation problems inasmuch as this item may have been incomprehensible to Iranian subjects. Therefore, it seems advisable that this item be removed from the Farsi version of the SSAS.

The test-retest consistency of the 10-item SSAS was evaluated by giving the scale to 30 of the same patients with functional gastrointestinal disorders after an interval of one month. There was a positive correlation of 0.80 between the total scores of the 2 tests. This finding is consistent with the results of an original study by Barsky et al. (2) in patients referring to a general hospital. The authors reported the test-retest reliability after an interval of between one and 6 weeks $(r=0.79)$. In another study, Gulec and Sayar (18) reported a test-retest correlation of 0.73 in healthy university students after an interval of one month. Based on these findings, the Farsi version of the SSAS seems to have good reliability.

The evaluation of the convergent validity with somatization was conducted by giving the patients the MSPQ and the SCL-90-R som subscale. As was expected, there were correlations between the total scores on the MSPQ, 
the SCL-90-R som subscale, and the total scores on the SSAS. The results indicated that these correlations were moderate by the SCL-90-R som $(\mathrm{r}=0.360, \mathrm{P}<0.05)$ and MSPQ $(r=0.522, \mathrm{P}<0.001)$. While Wise and Mann (20) posited that there is a link between somatization and amplification independent of depression and anxiety, Aronson et al. (15) demonstrated that the SSAS is more likely to be an index of negative emotionality and general distress than a valid measure of somatic sensitivity. In the present study, we also evaluated somatization using a different questionnaire (i.e. the MSPQ), which measures somatic arousal allied to psychological responses such as anxiety or depression. The positive correlation between the SSAS and the MSPQ in our study suggests that the SSAS is not independent of psychological responses, although it is a reliable indicator of somatization.

Finally, our assessment of the discriminant validity showed no significant difference between the patients and the control group. While Barsky et al. (2) specified that the SSAS is only suitable for medical outpatients, some studies have demonstrated the validity and reliability of the SSAS in non-clinical populations $(14,15,18)$. Our results suggest that the SSAS is not specific to patient populations, although we did not examine its psychometric properties in our healthy group.

Several limitations in the current study need to be addressed. First, with respect to the discriminant validity, the patient and control groups were not matched regarding their demographic characteristics. Second, the validity and reliability of the SSAS were not evaluated in the healthy group for comparison with the patients. Third, the test-retest reliability of the SSAS was examined in a limited population of patients with functional gastrointestinal disorders. Accordingly, caution should be exercised in the generalization of our findings.

Further research is needed to examine cross-cultural differences and to compare men and women in their amplification of somatic information. Also, the SSAS should be evaluated in various clinical populations such as people suffering from psychological problems (e.g. depression, anxiety, and somatoform disorders), patients with various functional somatic syndromes, and patients with serious medical diseases (e.g. chronic pains and infections) as well as in non-clinical populations in Iran.

Our results demonstrated that the SSAS is valid and reliable for patients with functional gastrointestinal disorders in the Iranian population.

A single factor model is the best model for the evaluation of somatosensory amplification and the Farsi version of the SSAS seems to assess the same construct as the original scale. This scale is an appropriate indicator of somatization and is not specific to patient populations.

\section{Authors' Contributions}

Alireza Aghayousefi conceived and designed the evaluation and helped to draft the manuscript. Narges Moham- madi participated in designing the evaluation, drafted the manuscript, helped to collect the clinical data, interpreted the clinical data, and performed parts of the statistical analysis. Mohammad Oraki revised the manuscript. Valiyollah Farzad performed the statistical analysis. Hammed Daghaghzadeh collected the clinical data and helped to interpret them. All authors read and approved the final manuscript.

\section{Declaration of Interest}

None declared.

\section{References}

1. Barsky AJ, Goodson JD, Lane RS, Cleary PD. The amplification of somatic symptoms. Psychosom Med. 1988;50(5):510-9.

2. Barsky AJ, Wyshak G, Klerman GL. The somatosensory amplification scale and its relationship to hypochondriasis.J Psychiatr Res. 1990;24(4):323-34.

3. Duddu V, Isaac MK, Chaturvedi SK. Somatization, somatosensory amplification, attribution styles and illness behaviour: a review. Int Rev Psychiatry. 2006;18(1):25-33.

4. Barsky AJ, Wyshak G. Hypochondriasis and somatosensory amplification. Br JPsychiatry. 1990;157(3):404-9.

5. Barsky AJ. Hypochondriasis: modern perspectives on an ancient malady. In: Starcevic V, Lipsitt DR, editors. Somatosensory amplification and hypochondriasis. Oxford, UK: Oxford University Press; 2011. pp. 223-48.

6. Nakao M, Barsky AJ. Clinical application of somatosensory amplification in psychosomatic medicine. Biopsychosoc Med. 2007;1:17.

7. Ak I, Sayar K, Yontem T. Alexithymia, somatosensory amplification and counter-dependency in patients with chronic pain. The Pain Clinic. 2004;16(1):43-51.

8. Kosturek A, Gregory RJ, Sousou AJ, Trief P. Alexithymia and somatic amplification in chronic pain. Psychosom. 1998;39(5):399-404.

9. Muramatsu K, Miyaoka H, Muramatsu Y, Fuse K, Yoshimine F, Kamijima K, et al. The amplification of somatic symptoms in upper respiratory tract infections. Gen Hosp Psychiatry. 2002;24(3):172-5.

10. Yavuz BG, Aydinlar EI, Dikmen PY, Incesu C. Association between somatic amplification, anxiety, depression, stress and migraine. J Headache Pain. 2013;14:53.

11. Kara B, Ozenli Y, Yalaki S. Exaggeration of physical symptoms in inflammatory bowel disease: Relation to depression, anxiety and quality of life. European Crohn's and Colitis Organisation; 2014 Available from: http://www.ecco-ibd.eu/publications/congressabstract-s/abstracts-2014/item/p627.

12. Jones MP, Roth LM, Crowell MD. Symptom reporting by functional dyspeptics during the water load test. Am J Gastroenterol. 2005;100(6):1334-9.

13. Jones MP, Schettler A, Olden K, Crowell MD. Alexithymia and somatosensory amplification in functional dyspepsia. Psychosomatics. 2004;45(6):508-16.

14. Bridou M, Aguerre C. Validity of the French form of the somatosensory amplification scale in a non-clinical sample. Health Psychol Res. 2013;1(e11):38-43.

15. Aronson KR, Barrett LF, Quigley KS. Feeling your body or feeling badly: evidence for the limited validity of the Somatosensory Amplification Scale as an index of somatic sensitivity. J Psychosom Res. 2001;51(1):387-94.

16. Fabbri S, Kapur N, Wells A, Creed F. Emotional, cognitive, and behavioral characteristics of medical outpatients: a preliminary analysis. Psychosomatics. 2001;42(1):74-7.

17. Lovas DA, Barsky AJ. Mindfulness-based cognitive therapy for hypochondriasis, or severe health anxiety: a pilot study.J Anxiety Disord. 2010;24(8):931-5.

18. Gulec H, Sayar K. Reliability and validity of the Turkish form of the Somatosensory Amplification Scale. Psychiatry Clin Neurosci. 2007;61(1):25-30.

19. Spinhoven P, van der Does AJ. Somatization and somatosensory 
amplification in psychiatric outpatients: an explorative study. Compr Psychiatry. 1997;38(2):93-7.

20. Wise TN, Mann LS. The relationship between somatosensory amplification, alexithymia, and neuroticism. J Psychosom Res. 1994;38(6):515-21.

21. Anisi J, Akbari F, Majdian M, Atashkar M, Ghorbani Z. [Standardization of mental disorders Symptoms Checklist 90 Revised (SCL-90 -R) in Army Staffs].J Mil Psychol . 2011;2(5):29-37.

22. Main CJ. The Modified Somatic Perception Questionnaire (MSPQ).J Psychosom Res. 1983;27(6):503-14

23. Jansson-Frojmark M, MacDonald S. Exploratory factor analysis of the Modified Somatic Perception Questionnaire on a sample with insomnia symptoms. Psychol Health Med. 2009;14(1):62-72.
24. Licciardone JC, Gatchel RJ, Kearns CM, Minotti DE. Depression, somatization, and somatic dysfunction in patients with nonspecific chronic low back pain: results from the OSTEOPATHIC Trial. JAm Osteopath Assoc. 2012;112(12):783-91.

25. Kako Joybari AA, Mohammadi N, Daghaghzadeh H, Ali Akbari $M$. [The relationship between emotional intelligence and somatization in patients with functional gastrointestinal disorders (FGID)]. Govaresh . 2014;19(4):250-6.

26. Speckens AE, Spinhoven P, Sloekers PP, Bolk JH, van Hemert AM. A validation study of the Whitely Index, the Illness Attitude Scales, and the Somatosensory Amplification Scale in general medical and general practice patients. J Psychosom Res. 1996;40(1):95-104. 